\title{
Cobalt sandwich complex-based covalent organic frameworks for chemical fixation of $\mathrm{CO}_{2}$
}

\author{
Yang $\mathrm{Li}^{1,2,3}$, Xiaoyu Song ${ }^{3}$, Guang Zhang ${ }^{3}$, Weihua $\mathrm{Chen}^{4}$, Lei Wang ${ }^{1,2}$, Yi Liu ${ }^{1 *}$ and Long $\mathrm{Chen}^{3^{*}}$
}

\begin{abstract}
Carbon dioxide as a notorious greenhouse gas triggers severe global warming which is threatening the balance of ecosystem. In this respect, effectively capturing and transforming $\mathrm{CO}_{2}$ into value-added chemicals are essential but still challenging tasks. As a kind of emerging crystalline porous material, covalent organic frameworks (COFs) have been demonstrated to be able to adsorb gases and function as catalysts to facilitate chemical transformations. Herein, we report an imine-linked, cobalt sandwich complex-based COF (Co-BD-COF) with high crystallinity and large surface area. Co-BD-COF can efficiently catalyze the transformation of $\mathrm{CO}_{2}$ into cyclic carbonates due to abundant metal sites and high porosity. In addition, Co-BD-COF exhibits high catalytic selectivity toward small ethylene oxide derivatives in cycloaddition reaction due to the large steric hindrance around the cobalt complexes rendered by the peripheral phenyl moieties. This new metal sandwich-type building block provides a new strategy for improving catalytic selectivity of COFs.
\end{abstract}

Keywords: covalent organic frameworks, cobalt sandwich complex, carbon dioxide, heterocatalysis, catalytic selectivity

\section{INTRODUCTION}

Excessive emission of carbon dioxide has been a persistent environment issue due to the rapid development of industry and massive combustion of fossil fuels, which, however, has triggered huge threats to the whole world, such as global warming and the accompanied collapse of ecosystem. In this context, effectively reducing the concentration of $\mathrm{CO}_{2}$ in the atmosphere is a critical and challenging task to be solved [1]. For carbon dioxide capture and utilization technology, capturing $\mathrm{CO}_{2}$ and using it as a green $\mathrm{C} 1$ chemical raw material to produce value-added chemicals are sustainable methods to reduce $\mathrm{CO}_{2}$ in the atmosphere and achieve a carbon-neutral energy cycle [2-6]. Cyclic carbonates are atom-economic non-reducing products; especially, cyclic carbonates substituted with shorter alkyl chains have many advantages in the electrolytes of lithium-ion batteries, such as high chemical stability and wide temperature range [7]. Various kinds of materials including ionic liquids [8], ammonium salts [9], metal-organic frameworks (MOFs) [10-12], and covalent organic frameworks (COFs) [13] have been developed for effi- cient conversion of $\mathrm{CO}_{2}$ into cyclic carbonates.

MOFs and COFs are heterogeneous catalysts [14], which can realize rapid recovery of the catalyst and inhibit the occurrence of polymerization in the reaction of catalyzing cyclocarbonate. Functional MOFs are ideal candidates for the chemical fixation of carbon dioxide, due to the active metal sites for gas adsorption and catalysis. However, the limited stability of MOFs inhibits their pratical catalysis applications [10]. COFs represent an emerging class of crystalline porous materials featuring high stability and regular pore channels [15-18]. Although some COFs have already been used as heterogeneous catalysts in the chemical conversion of $\mathrm{CO}_{2}[13,19,20]$, there still exists demands of building more effective COFs to control the product selectivity toward improving atom utilization and reduce purification costs during production. Selective catalysis can be achieved by reducing the pore size [21], but the effect of this strategy is not significant for improving the selectivity of COFs for $\mathrm{CO}_{2}$ fixation $[22,23]$. Therefore, in order to achieve high catalytic performance and selectivity for $\mathrm{CO}_{2}$ fixation, we expect to introduce sandwich-type metal complexes containing bulky organic ligands as selective catalytic centers between the layers of COFs to avoid the limitation of pore size [24]. Additionally, the unlimited pore size of COFs can facilitate mass transfer in catalytic reactions [25].

In this work, a metallocene analogous cobalt sandwich complex $\left[\left(\eta^{5}\right.\right.$-cyclopentadienyl $)\left(\eta^{4}\right.$-cyclobutadiene $)$ cobalt $]\left(\left(\eta^{5}-\mathrm{Cp}\right)\right.$ $\left.\mathrm{Co}\left(\eta^{4}-\mathrm{C}_{4} \mathrm{Ph}_{4}\right)\right)$ was employed as the building block to construct a metal-containing COF (Co-BD-COF). ( $\left.\eta^{5}-\mathrm{Cp}\right) \mathrm{Co}\left(\eta^{4}-\mathrm{C}_{4} \mathrm{Ph}_{4}\right)$ obeys the Hückel's rule and exhibits high stability under air and moisture [26-28]. The cobalt complexes within the skeleton of the COF exist in the form of metallocene with a relatively large basal bulkiness, which might render enhanced catalytic selectivity due to the space confinement effects around Co centers. For the synthesis of Co-BD-COF, the aldehyde monomer (Co$\mathrm{CHO}$ ) was synthesized firstly (Scheme S1 and Figs S1-S3). Furthermore, linear and long benzidine (4,4'-biphenylenediamine, BD) was selected as the amino-functionalized monomer (Scheme 1). Two-dimensional (2D) crystalline Co-BD-COF was prepared by a Schiff base polycondensation between $\mathrm{Co}-\mathrm{CHO}$ and $\mathrm{BD}$, in which cobalt atoms were regularly arranged and precisely positioned within the $2 \mathrm{D}$ layers [15]. In addition, the uniformly dispersed Co within the framework of COF promised

\footnotetext{
${ }^{1}$ Shenzhen Key Laboratory of Polymer Science and Technology, Guangdong Research Center for Interfacial Engineering of Functional Materials, College of Materials Science and Engineering, Shenzhen University, Shenzhen 518060, China

${ }^{2}$ College of Physics and Optoelectronic Engineering, Shenzhen University, Shenzhen 518060, China

${ }^{3}$ Tianjin Key Laboratory of Molecular Optoelectronic Science, Department of Chemistry, Tianjin University, Tianjin 300072, China

${ }^{4}$ College of Chemistry and Green Catalysis Center, Zhengzhou University, Zhengzhou 450001, China

* Corresponding authors (emails: liuyiacee@szu.edu.cn (Liu Y); long.chen@tju.edu.cn (Chen L))
} 


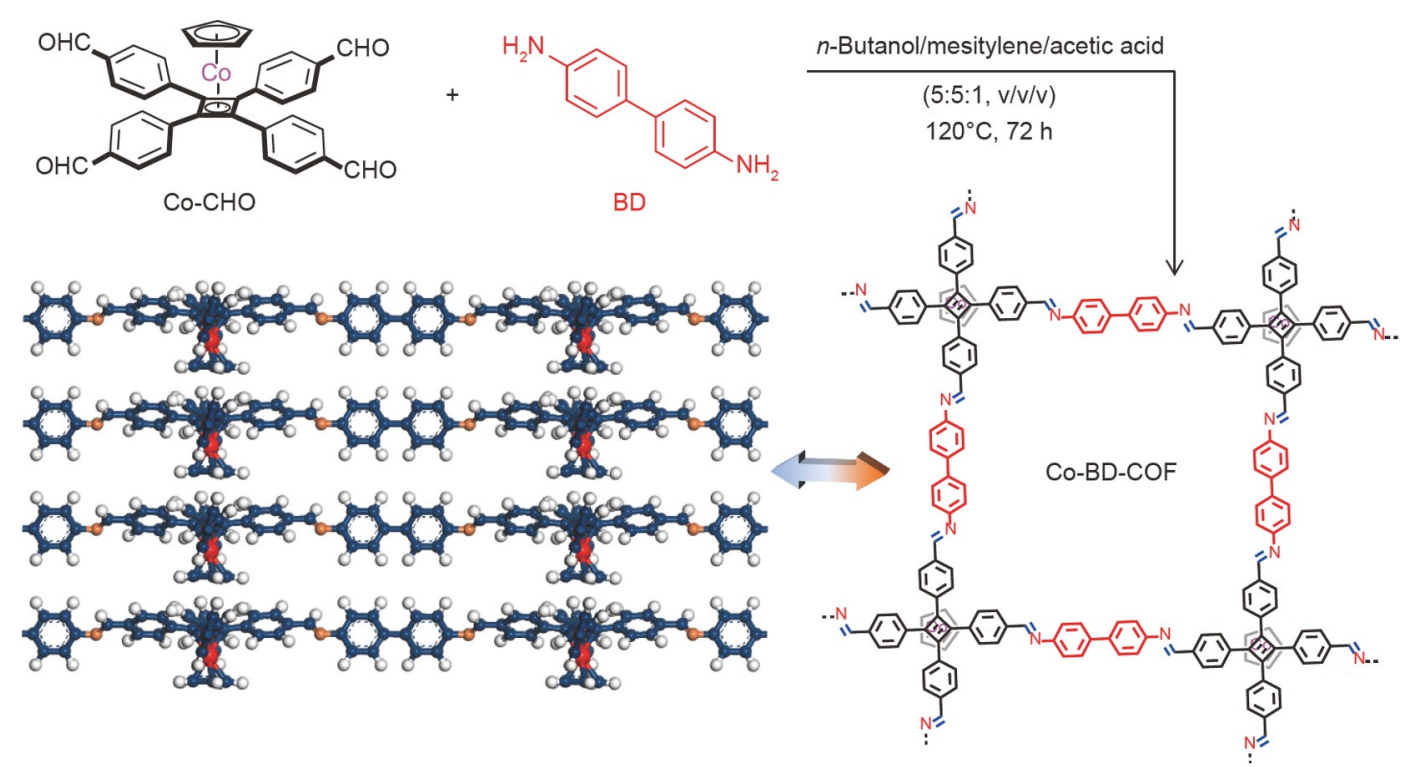

Scheme 1 Synthetic route and stacking mode of Co-BD-COF.

recyclable catalytic activity of the Co-BD-COF by effectively preventing the aggregation among Co atoms. Interestingly, the catalytic activity of COFs decreased sharply upon increasing the size of the substrates, indicating that COFs had a high selectivity to the molecular size of the substrate. These results suggest that sandwich-type COFs containing metal may serve as an excellent material for $\mathrm{CO}_{2}$ fixation application due to their high efficiency and size selectivity.

\section{EXPERIMENTAL SECTION}

\section{Preparation of Co-BD-COF}

Co-CHO (12.1 mg, $0.02 \mathrm{mmol})$ and BD (7.36 mg, $0.04 \mathrm{mmol})$ were added to a $10-\mathrm{mL}$ glass tube, and then $1.1 \mathrm{~mL}$ of a $5: 5: 1(\mathrm{v} /$ $v / v)$ solution of $n$-butanol/mesitylene/acetic acid $\left(6 \mathrm{~mol} \mathrm{~L}^{-1}\right)$ was added. The tube was degassed under vacuum at $77 \mathrm{~K}$ and sealed. Subsequently, the tube was heated at $120^{\circ} \mathrm{C}$ and kept for three days. After cooling to room temperature, the product was isolated by filtration and washed with tetrahydrofuran and acetone, and dried in vacuum, affording an orange powder in $89 \%$ yield.

\section{Catalytic $\mathrm{CO}_{2}$ cycloaddition with epoxides to produce cyclic carbonates}

In a typical reaction, the catalyst (COF, $18 \mathrm{mg}, 2 \mathrm{~mol} \%$ ), tetrabutylammonium bromide (TBAB, $0.05 \mathrm{mmol}$ ) and epoxides ( $1 \mathrm{mmol})$ were taken in a pre-dried Schlenk tube. After sealing and purging with $\mathrm{CO}_{2}(1 \mathrm{~atm})$, the Schlenk tube was placed in a preheated oil bath and stirred for $24 \mathrm{~h}$ at $40^{\circ} \mathrm{C}$. After the reaction, the tube was cooled to room temperature. The reaction mixture was filtered off and washed thoroughly with dichloromethane to ensure complete extraction of the product. The collected filtrate contained both the product and co-catalyst (TBAB) which could be removed by washing with water. The remaining filtrate was evaporated under reduced pressure to obtain the product. Then the samples were analyzed by ${ }^{1} \mathrm{H}$ nuclear magnetic resonance $\left({ }^{1} \mathrm{H}\right.$ NMR) spectroscopy. The recovered catalyst was dried under vacuum and reused for next cycles.

\section{RESULTS AND DISCUSSION}

\section{Structure and physical characterization}

Co-BD-COF was synthesized by solvothermal polycondensation of the monomers at $120^{\circ} \mathrm{C}$ in a mixed solvent of $n$-butanol/ mesitylene/acetic acid $\left(6 \mathrm{~mol} \mathrm{~L}^{-1}\right)$ for three days [29]. The crystallinity of Co-BD-COF was confirmed by powder X-ray diffraction (PXRD) analysis and the extended architecture of Co-BD-COF was modeled (Table S1). The experimental PXRD pattern of Co-BD-COF displayed an intense diffraction peak at $3.70^{\circ}$ and two other signals at $6.55^{\circ}$ and $11.00^{\circ}$ (Fig. 1a), which were assigned to the (100), (200) and (300) facets, respectively. Co-BD-COF exhibited an eclipsed AA (Fig. 1b, c) rather than a staggered $\mathrm{AB}$ stacking mode (Fig. S4) by comparing the experimental PXRD patterns and the simulated results. Pawley refinement results indicated that the simulated structure matched well with the experimental data $\left(R_{\mathrm{wp}}=6.70 \%\right.$ and $R_{\mathrm{p}}=$ $5.02 \%)$. Interestingly, the metal atoms were uniformly located between rather than within the layers (Fig. 1c, d).

Fourier transform infrared (FT-IR) spectrum of the Co-BDCOF showed the characteristic peak for $\mathrm{C}=\mathrm{N}$ linkages at $1624 \mathrm{~cm}^{-1}$, significant disappearance of $\mathrm{C}=\mathrm{O}\left(1692 \mathrm{~cm}^{-1}\right)$ and the $\mathrm{N}-\mathrm{H}$ stretching vibrations (3439 and $3329 \mathrm{~cm}^{-1}$ ) from the precursors, which indicated successful imine condensation (Fig. S5) [30,31]. To further verify the chemical structure of the $\mathrm{COF}$, a model compound (Co-CHOA) was synthesized through a Schiff base reaction between Co-CHO and aniline (Scheme S2 and Figs S6-S8). Similarly, the same characteristic $C=N$ stretching band at $1624 \mathrm{~cm}^{-1}$ was observed for Co-CHOA (Fig. S5a) [32], which further proved that Co-BD-COF was connected by imine linkages. The chemical structure of Co-BD$\mathrm{COF}$ was further characterized by the solid-state ${ }^{13} \mathrm{C}$ cross polarization magic angle spinning (CP-MAS) NMR spectra. The characteristic signal at $158 \mathrm{ppm}$ and significant attenuation of the signal of aldehyde carbon around $190 \mathrm{ppm}$ for Co-BD-COF further confirmed the formation of $\mathrm{C}=\mathrm{N}$ linkages (Fig. S9) [33], whereas the ${ }^{13} \mathrm{C}$ CP-MAS NMR spectrum of Co-BD-COF displayed the carbon signals of cyclobutadiene $(\mathrm{Cb})$ and cyclo- 

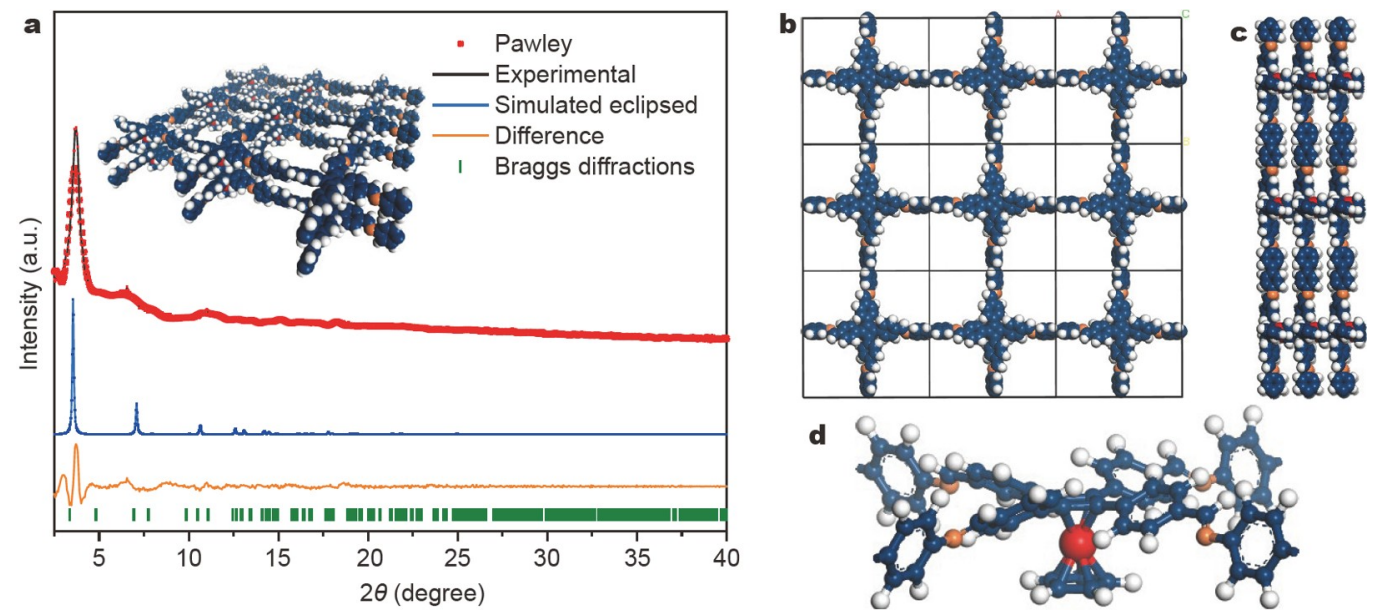

Figure 1 (a) Experimental PXRD pattern (black) and Pawley refinement (red) of Co-BD-COF, their difference (orange), simulated profiles using the eclipsed AA arrangement (blue) and braggs diffractions stacking modes (green). Top view (b) and side view (c) of the AA stacking modes for Co-BD-COF. (d) Side view of a unit cell of Co-BD-COF.

pentadienyl (Cp) moieties at 73 and 83 ppm, respectively [28]. In addition, the characteristic carbon signals of Co-BD-COF were consistent with those of Co-CHOA (Fig. S9). Moreover, elemental analysis of Co-BD-COF revealed that the contents of $\mathrm{C}$, $\mathrm{H}$ and $\mathrm{N}$ were in good agreement with the theoretical values of $\mathrm{C}_{61} \mathrm{H}_{41} \mathrm{CoN}_{4}$ for Co-BD-COF (Table S2). The content of Co atoms in Co-BD-COF was determined to be up to $7.51 \mathrm{wt} \%$ obtained from inductively coupled plasma optical emission spectrometry (ICP-OES, Table S3).

The morphology of Co-BD-COF was characterized through field emission scanning electron microscopy (FE-SEM). The images revealed Co-BD-COF appeared as lamellar particles (Fig. S10). More detailed characterization was implemented by using transmission electron microscopy (TEM). The images unveiled that the lamellar particles were composed of stacked COF layers (Fig. S11). Further elemental mapping analysis indicated a homogeneous distribution of $\mathrm{C}$ and Co elements on the nanosheets of Co-BD-COF. Thermogravimetric analysis indicated that $\mathrm{Co}-\mathrm{BD}-\mathrm{COF}$ was thermally stable up to $472^{\circ} \mathrm{C}$ (loss 5\%) under nitrogen atmosphere (Fig. S12).

\section{Porosity analysis}

The permanent porosity of the Co-BD-COF was assessed by $\mathrm{N}_{2}$ adsorption/desorption measurements at $77 \mathrm{~K}$. As displayed in Fig. S13a, the $\mathrm{N}_{2}$ sorption isotherms for Co-BD-COF suggested type IV characteristics, which indicated Co-BD-COF possesses mesopores. The Brunauer-Emmett-Teller (BET) surface area of Co-BD-COF was determined to be $1040 \mathrm{~m}^{2} \mathrm{~g}^{-1}$. The pore size distribution of Co-BD-COF indicated a uniform pore size of $2.0 \mathrm{~nm}$, which was simulated by the nonlocal density functional theory (NLDFT, Fig. S13b). The result was close to the $d$-spacing value as calculated from the PXRD pattern.

\section{Chemical fixation of $\mathrm{CO}_{2}$}

Metallosalen (M-Salen) complex has been utilized to catalyze the cycloaddition reaction between carbon dioxide and epoxides to form cyclic carbonates, which has recently received increasing research interest [34]. Considering the porous architecture and high specific surface area of Co-BD-COF, the abundant Co sites anchored in the open channels of Co-BD-COF could accommodate guest molecules (e.g., $\mathrm{CO}_{2}$ ) and serve as active sites to mediate catalytic reactions [15,35]. To evaluate the $\mathrm{CO}_{2}$ adsorption capacity of $\mathrm{Co}-\mathrm{BD}-\mathrm{COF}, \mathrm{CO}_{2}$ sorption isotherms were recorded at $273 \mathrm{~K}$ and the maximum uptake of $\mathrm{CO}_{2}$ was found to be $1.17 \mathrm{mmol} \mathrm{g}^{-1}$ (Fig. S14). Therefore, we expected Co-BD-COF could function as a potential heterogeneous catalyst for cycloaddition reaction between epoxides and $\mathrm{CO}_{2}$ [36]. Furthermore, Co-BD-COF maintained high thermal stability after being heated in $\mathrm{CO}_{2}$ atmosphere for two days, as evidenced from the PXRD patterns of the COF samples (Fig. S15).

In order to systematically investigate the catalytic properties of Co-BD-COF, different epoxide derivatives were used as substrates in the cycloaddition reaction. Typically, all reactions were carried out at $40^{\circ} \mathrm{C}$ with a balloon of $\mathrm{CO}_{2}(0.1 \mathrm{MPa})$ and $\mathrm{TBAB}$ as the co-catalyst (Table 1) [13]. The yields of the generated cyclic carbonates were calculated based on the ${ }^{1} \mathrm{H}$ NMR spectroscopy. The cycloaddition reaction between $\mathrm{CO}_{2}$ and epichlorohydrin was selected as a model reaction for comparison (Table S4), which achieved a yield of 95\%. It disclosed that the yields were reduced to $36 \%$ and $9 \%$, respectively, when Co-BD$\mathrm{COF}$ or TBAB was absent. This indicated that both the COF and $\mathrm{TBAB}$ played indispensable roles in achieving high catalytic performance.

As summarized in Table 1, for 1,2-epoxybutane and 1,2epoxypentane, the yields of cyclic carbonates reached $99 \%$ and $94 \%$, respectively (entries 1 and 2). With elongating the alkyl chains, the yields decreased accordingly; for example, the yield dropped to $83 \%$ for 1,2-epoxyhexane (entry 3) and was further reduced to $43 \%$ for allyl glycidyl ether (entry 4 ). In general, the ethylene oxide derivatives with short alkyl chains could afford very high yields in this catalytic reaction. The high catalytic selectivity of Co-BD-COF toward less bulky epoxides could be ascribed to the special sandwich structures of the cobalt complexes in Co-BD-COF (Fig. 1d). This trend of catalytic selectivity was also observed for halogen-substituted substrates. For instance, the yield was $95 \%$ for chloropropene carbonate and was dropped to $82 \%$ for bromopropylene carbonate (entries 5 and 6). In addition, when more sterically hindered aromatic epoxides, i.e. styrene oxide and glycidyl phenyl ether were investigated, very low yields of ca. $11 \%$ and $32 \%$ were obtained, respectively (entries 7 and 8), probably due to the bulkiness of the substrates (Fig. S16) [37]. Moreover, it showed that the steric 
Table 1 Catalytic cycloaddition of $\mathrm{CO}_{2}$ with different epoxide substrates using $\mathrm{Co}-\mathrm{BD}-\mathrm{COF}$ as the catalyst

$$
\underset{\mathrm{O}}{\mathrm{O}}+\mathrm{CO}_{2} \underset{\mathrm{TBAB}}{\stackrel{\mathrm{CO}-\mathrm{BD}-\mathrm{COF}}{\longrightarrow}} \underset{\mathrm{O}}{\mathrm{O}} y^{\mathrm{R}}
$$

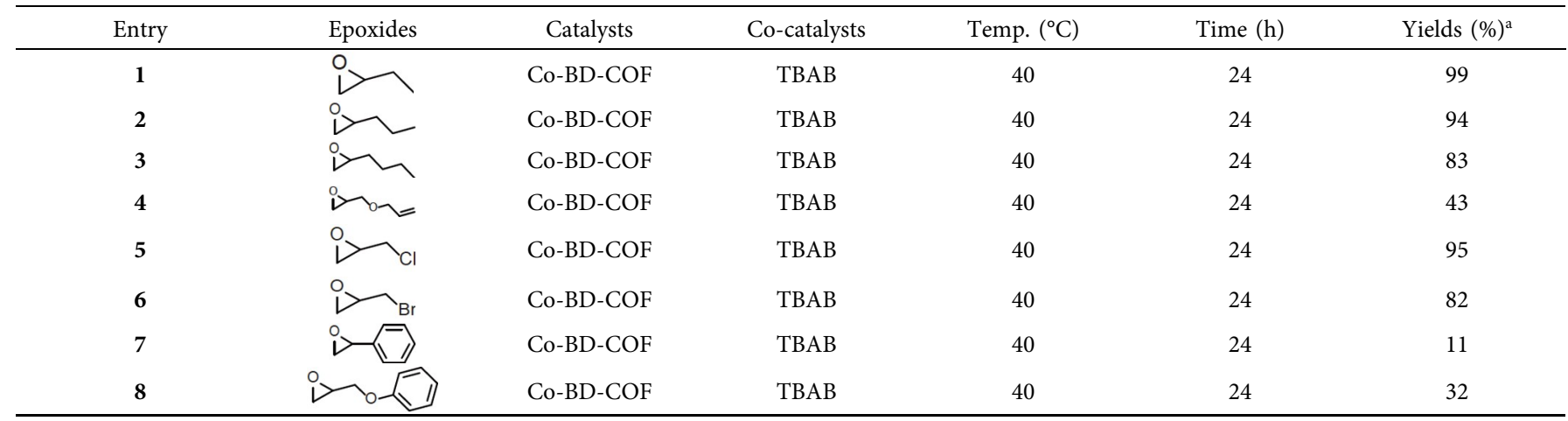

a) Determined by ${ }^{1} \mathrm{H}$ NMR spectroscopic analysis.
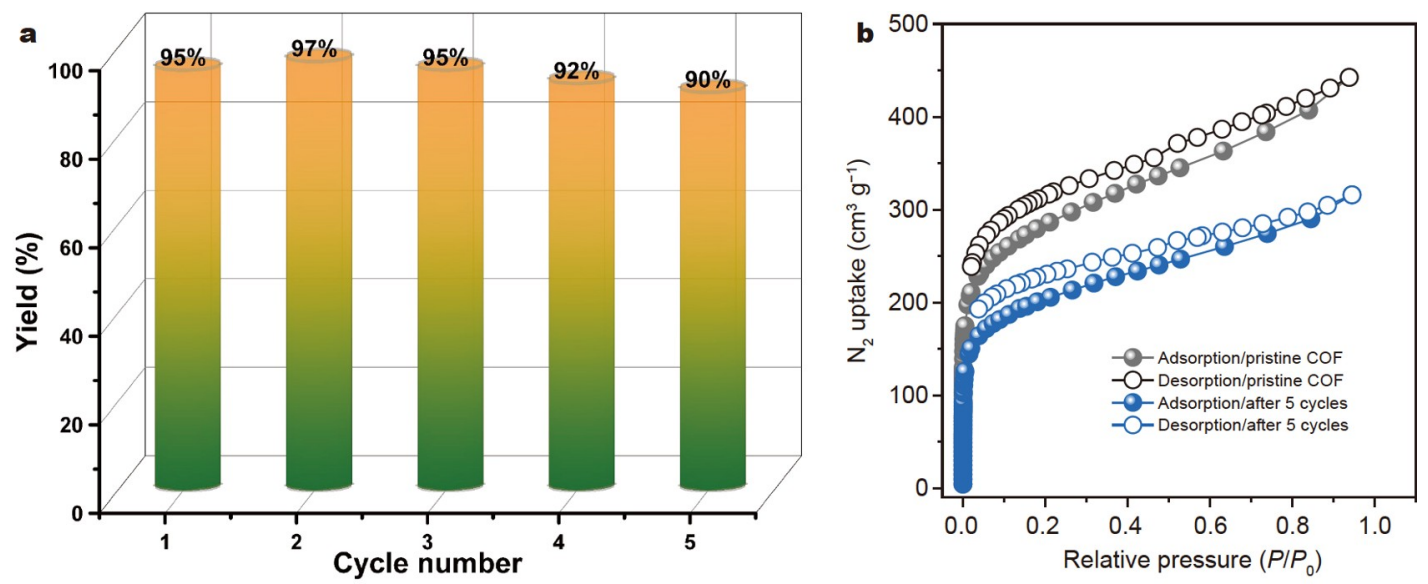

Figure 2 (a) Recycling catalytic test of Co-BD-COF for the $\mathrm{CO}_{2}$ cycloaddition with epichlorohydrin. (b) $\mathrm{N}_{2}$ adsorption/desorption isotherms of Co-BD-COF before (black) and after (blue) the 5th run of cyclic reactions.

hindrance effect of aromatic rings was higher than that of chain substituents by comparing entries 4 and 8 . Consequently, CoBD-COF was demonstrated to exhibit high catalytic activity and selectivity toward small-size epoxides with low steric hindrance for effective chemical fixation of $\mathrm{CO}_{2}$ [15].

To further evaluate the reusability of Co-BD-COF, the model reaction using epichlorohydrin as the substrate was studied $[38,39]$. The results showed that the yield of chloropropene carbonate remained $95 \%$ after three catalytic cycles and still maintained $90 \%$ after five repeated catalytic cycles (Fig. 2a and Table S5). In addition, the PXRD pattern and nitrogen sorption isotherms of Co-BD-COF were evaluated again after the catalytic cycles, and little changes in both crystallinity and specific surface area of Co-BD-COF were observed before and after the catalytic cycles (Fig. 2b and Fig. S17). In addition, the content of Co element in Co-BD-COF was measured again after the catalytic cycles and showed negligible change, which further proved the metal sites within the COF were intact during the catalytic cycles (Table S3). Thus, Co-BD-COF manifested high catalytic performance with good reusability toward $\mathrm{CO}_{2}$ fixation [19,40-42].

To shed more lights into the effect of Co-BD-COF catalyst on the cycloaddition reaction between $\mathrm{CO}_{2}$ and epoxides, a rea- sonable mechanism is proposed by referring to the literature (Fig. 3) [15]. Firstly, the Co sandwich complexes interact with the oxygen atoms of the epoxides. The sandwich structure of $\left(\eta^{5}-\mathrm{Cp}\right) \mathrm{Co}\left(\eta^{4}-\mathrm{C}_{4} \mathrm{Ph}_{4}\right)$ can prevent the bulky epoxides from interacting with the metal site. Next, a bromide from TBAB attacks one carbon atom of epoxide group from the less sterically-hindered side to form an oxyanion intermediate. Subsequently, the oxyanion attacks one $\mathrm{CO}_{2}$ molecule to form an alkyl carbonate anion intermediate, which is followed by a cyclizing process to form the final carbonate and a release of Co-BD-COF for the next catalytic cycle $[13,40]$.

\section{CONCLUSIONS}

An unprecedented cobalt sandwich complex-based imine COF (Co-BD-COF) was successfully developed, which showed high crystallinity, large surface area, and good stability. The metal active sites uniformly arranged in the Co-BD-COF skeleton could serve as the main catalytic centers to mediate effective fixation of $\mathrm{CO}_{2}$ by cycloaddition reaction of $\mathrm{CO}_{2}$ with epoxides in high yields. Meanwhile, Co-BD-COF as a heterogeneous catalyst was highly recyclable and exhibited superior activity and excellent selectivity toward small epoxide substrates with low 


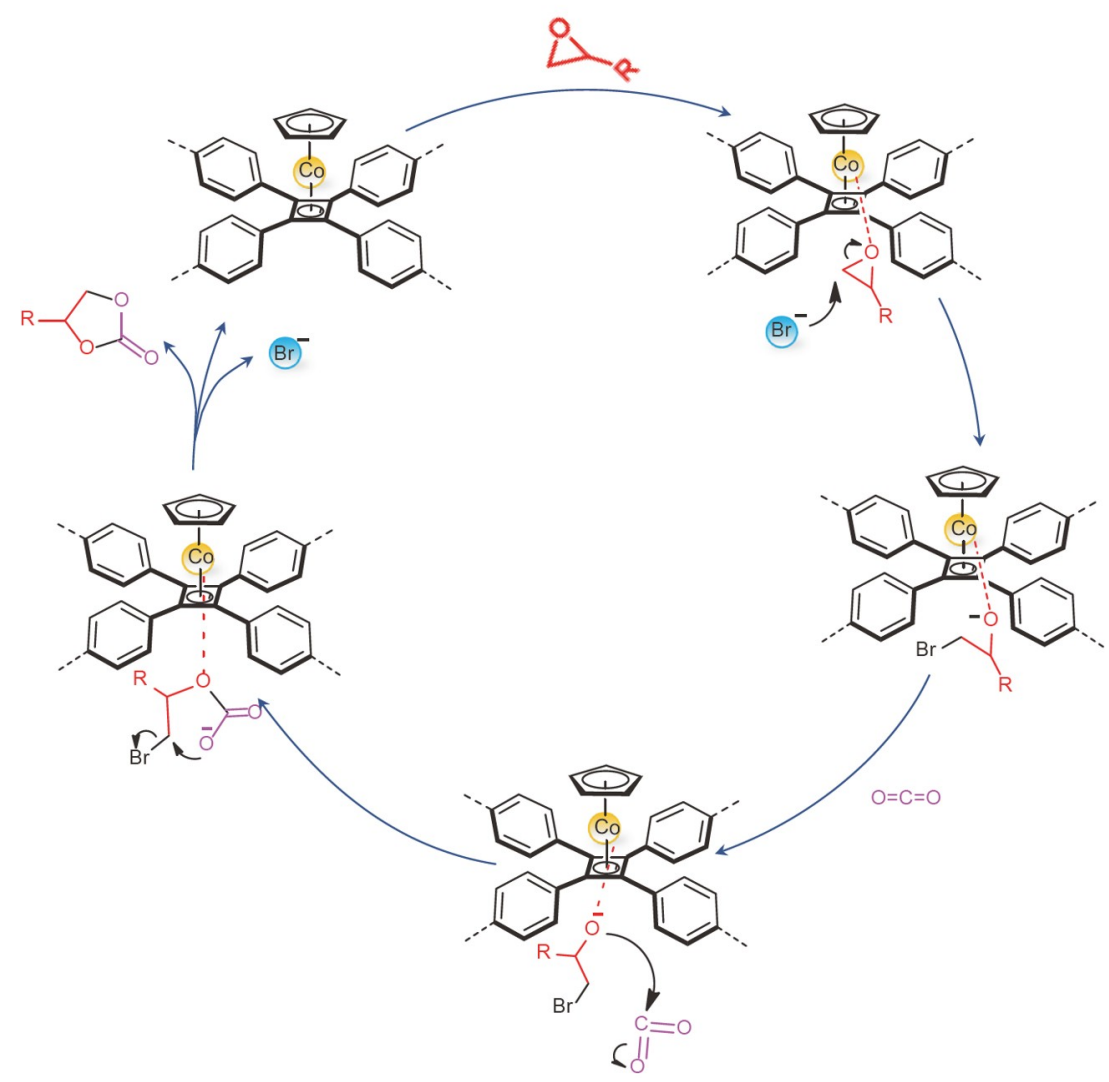

Figure 3 Proposed mechanism for the cycloaddition of $\mathrm{CO}_{2}$ with epoxides catalyzed by Co-BD-COF.

steric hindrance. The current work not only enriches the diversity of metal-functionalized COFs, but also provides new insights into the design of COFs as effective heterogeneous catalysts.

\section{Received 13 September 2021; accepted 22 October 2021;} published online 17 December 2021

1 Liu J, Thallapally PK, McGrail BP, et al. Progress in adsorption-based $\mathrm{CO}_{2}$ capture by metal-organic frameworks. Chem Soc Rev, 2012, 41: 2308-2322

2 Patel HA, Byun J, Yavuz CT. Carbon dioxide capture adsorbents: Chemistry and methods. ChemSusChem, 2017, 10: 1303-1317

3 Yi J, Si D, Xie R, et al. Conductive two-dimensional phthalocyaninebased metal-organic framework nanosheets for efficient electroreduction of $\mathrm{CO}_{2}$. Angew Chem Int Ed, 2021, 60: 17108-17114

$4 \mathrm{Wu} \mathrm{Q}, \mathrm{Xie} \mathrm{RK}, \mathrm{Mao} \mathrm{MJ}$, et al. Integration of strong electron transporter tetrathiafulvalene into metalloporphyrin-based covalent organic framework for highly efficient electroreduction of $\mathrm{CO}_{2}$. ACS Energy Lett, 2020, 5: 1005-1012

5 Zhang M, Si D, Yi J, et al. Conductive phthalocyaninebased covalent organic framework for highly efficient electroreduction of carbon dioxide. Small, 2020, 16: 2005254

6 Zhang MD, Si DH, Yi JD, et al. Conductive phthalocyanine-based metal-organic framework as a highly efficient electrocatalyst for carbon dioxide reduction reaction. Sci China Chem, 2021, 64: 1332-1339

7 Cheng XB, Yan C, Chen X, et al. Implantable solid electrolyte interphase in lithium-metal batteries. Chem, 2017, 2: 258-270

$8 \mathrm{Xu} \mathrm{BH}$, Wang JQ, Sun J, et al. Fixation of $\mathrm{CO}_{2}$ into cyclic carbonates catalyzed by ionic liquids: A multi-scale approach. Green Chem, 2015, 17: $108-122$

9 Cokoja M, Wilhelm ME, Anthofer MH, et al. Synthesis of cyclic carbonates from epoxides and carbon dioxide by using organocatalysts.
ChemSusChem, 2015, 8: 2436-2454

10 Miralda CM, Macias EE, Zhu M, et al. Zeolitic imidazole framework-8 catalysts in the conversion of $\mathrm{CO}_{2}$ to chloropropene carbonate. ACS Catal, 2011, 2: 180-183

11 Zou YH, Huang YB, Si DH, et al. Porous metal-organic framework liquids for enhanced $\mathrm{CO}_{2}$ adsorption and catalytic conversion. Angew Chem Int Ed, 2021, 60: 20915-20920

12 Liang J, Chen RP, Wang XY, et al. Postsynthetic ionization of an imidazole-containing metal-organic framework for the cycloaddition of carbon dioxide and epoxides. Chem Sci, 2017, 8: 1570-1575

13 Zhi Y, Shao P, Feng X, et al. Covalent organic frameworks: Efficient, metal-free, heterogeneous organocatalysts for chemical fixation of $\mathrm{CO}_{2}$ under mild conditions. J Mater Chem A, 2018, 6: 374-382

14 He C, Liang J, Zou YH, et al. Metal-organic frameworks bonded with metal $n$-heterocyclic carbenes for efficient catalysis. Natl Sci Rev, 2021, doi: $10.1093 /$ nsr/nwab157

15 Lohse MS, Bein T. Covalent organic frameworks: Structures, synthesis, and applications. Adv Funct Mater, 2018, 28: 1705553

16 Dong J, Han X, Liu Y, et al. Metal-covalent organic frameworks (MCOFs): A bridge between metal-organic frameworks and covalent organic frameworks. Angew Chem Int Ed, 2020, 59: 13722-13733

17 Segura JL, Royuela S, Mar Ramos M. Post-synthetic modification of covalent organic frameworks. Chem Soc Rev, 2019, 48: 3903-3945

$18 \mathrm{Wu}$ Q, Mao MJ, Wu QJ, et al. Construction of donor-acceptor heterojunctions in covalent organic framework for enhanced $\mathrm{CO}_{2}$ electroreduction. Small, 2021, 17: 2004933

19 Xu K, Dai Y, Ye B, et al. Two dimensional covalent organic framework materials for chemical fixation of carbon dioxide: Excellent repeatability and high selectivity. Dalton Trans, 2017, 46: 10780-10785

20 Saptal V, Shinde DB, Banerjee R, et al. State-of-the-art catechol porphyrin cof catalyst for chemical fixation of carbon dioxide via cyclic carbonates and oxazolidinones. Catal Sci Technol, 2016, 6: 6152-6158

21 Fang Q, Gu S, Zheng J, et al. 3D microporous base-functionalized covalent organic frameworks for size-selective catalysis. Angew Chem 
Int Ed, 2014, 53: 2878-2882

22 Liang J, Huang YB, Cao R. Metal-organic frameworks and porous organic polymers for sustainable fixation of carbon dioxide into cyclic carbonates. Coord Chem Rev, 2019, 378: 32-65

23 Puthiaraj P, Lee YR, Zhang S, et al. Triazine-based covalent organic polymers: Design, synthesis and applications in heterogeneous catalysis. J Mater Chem A, 2016, 4: 16288-16311

24 Bergin E, Hughes DL, Richards CJ. Application of the Suzuki reaction to the asymmetric desymmetrisation of 1,2- and 1,3-disubstituted bulky cobalt metallocenes. Tetrahedron-Asymmetry, 2010, 21: 1619-1623

25 Ding SY, Gao J, Wang Q, et al. Construction of covalent organic framework for catalysis: Pd/COF-LZU1 in Suzuki-Miyaura coupling reaction. J Am Chem Soc, 2011, 133: 19816-19822

26 Kumar D, Deb M, Singh J, et al. Chemistry of the highly stable hindered cobalt sandwich compound $\left(\eta^{5}-\mathrm{Cp}\right) \mathrm{Co}\left(\eta^{4}-\mathrm{C}_{4} \mathrm{Ph}_{4}\right)$ and its derivatives. Coord Chem Rev, 2016, 306: 115-170

27 Gan Q, Ronson TK, Vosburg DA, et al. Cooperative loading and release behavior of a metal-organic receptor. J Am Chem Soc, 2015, 137: 17701773

28 Singh N, Singh J, Kim D, et al. Coordination-driven self-assembly of heterotrimetallic barrel and bimetallic cages using a cobalt sandwichbased tetratopic donor. Inorg Chem, 2018, 57: 3521-3528

29 Li Y, Chen W, Gao R, et al. 2D covalent organic frameworks with builtin amide active sites for efficient heterogeneous catalysis. Chem Commun, 2019, 55: 14538-14541

30 Li Z, Feng X, Zou Y, et al. A 2D azine-linked covalent organic framework for gas storage applications. Chem Commun, 2014, 50: 1382513828

31 Han X, Huang J, Yuan C, et al. Chiral 3D covalent organic frameworks for high performance liquid chromatographic enantioseparation. J Am Chem Soc, 2018, 140: 892-895

32 Ascherl L, Sick T, Margraf JT, et al. Molecular docking sites designed for the generation of highly crystalline covalent organic frameworks. Nat Chem, 2016, 8: 310-316

33 Jin E, Geng K, Lee KH, et al. Topology-templated synthesis of crystalline porous covalent organic frameworks. Angew Chem Int Ed, 2020, 59: $12162-12169$

34 Zhou W, Deng QW, Ren GQ, et al. Enhanced carbon dioxide conversion at ambient conditions via a pore enrichment effect. Nat Commun, 2020, 11: 4481

35 Stegbauer L, Hahn MW, Jentys A, et al. Tunable water and $\mathrm{CO}_{2}$ sorption properties in isostructural azine-based covalent organic frameworks through polarity engineering. Chem Mater, 2015, 27: 78747881

36 Sun Q, Aguila B, Perman J, et al. Flexibility matters: Cooperative active sites in covalent organic framework and threaded ionic polymer. J Am Chem Soc, 2016, 138: 15790-15796

37 Gao WY, Tsai CY, Wojtas L, et al. Interpenetrating metal-metalloporphyrin framework for selective $\mathrm{CO}_{2}$ uptake and chemical transformation of $\mathrm{CO}_{2}$. Inorg Chem, 2016, 55: 7291-7294

38 Subramanian S, Park J, Byun J, et al. Highly efficient catalytic cyclic carbonate formation by pyridyl salicylimines. ACS Appl Mater Interfaces, 2018, 10: 9478-9484

$39 \mathrm{Xu} \mathrm{H}$, Zhai B, Cao CS, et al. A bifunctional europium-organic framework with chemical fixation of $\mathrm{CO}_{2}$ and luminescent detection of $\mathrm{Al}^{3+}$. Inorg Chem, 2016, 55: 9671-9676

40 Meng XL, Nie Y, Sun J, et al. Functionalized dicyandiamide-formaldehyde polymers as efficient heterogeneous catalysts for conversion of $\mathrm{CO}_{2}$ into organic carbonates. Green Chem, 2014, 16: 2771-2778

$41 \mathrm{Ma} \mathrm{H}$, Wang Z, Zhao YH, et al. Microporous polymer based on hexaazatriphenylene-fused triptycene for $\mathrm{CO}_{2}$ capture and conversion. Sci China Mater, 2019, 63: 429-436

42 Zhao D, Kong $\mathrm{C}, \mathrm{Du} \mathrm{H}$, et al. A molecular-templating strategy to polyamine-incorporated porous organic polymers for unprecedented $\mathrm{CO}_{2}$ capture and separation. Sci China Mater, 2019, 62: 448-454

Acknowledgements This work was supported by the National Natural Science Foundation of China (51973153 and 21704065), the National Key
Research and Development Program of China (2017YFA0207500), Guangdong Basic and Applied Basic Research Foundation (2021A1515010228), and the Innovation Research Foundation of Shenzhen (JCYJ20190808115215125).

Author contributions $\quad \mathrm{Li} \mathrm{Y}$ and Song $\mathrm{X}$ conducted the experiment; Zhang $\mathrm{G}$, Chen W and Wang L performed some data analysis and offered helpful suggestions; Liu Y and Chen L designed this work; Li Y, Song X, Zhang G, Liu $\mathrm{Y}$ and Chen $\mathrm{L}$ analyzed the data and wrote the paper. All authors contributed to the general discussion.

Conflict of interest The authors declare that they have no conflict of interest.

Supplementary information Experimental details and supporting data are available in the online version of the paper.

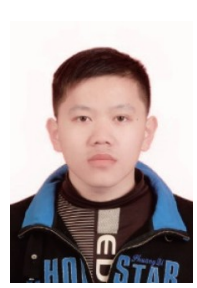

Yang Li received his $\mathrm{PhD}$ degree in 2020 at Tianjin University under the supervision of Prof. Long Chen, and afterward started to conduct his postdoc research at Shenzhen University. His current research focuses on the design and synthesis of $2 \mathrm{D}$ conjugated porous polymers for catalysis.

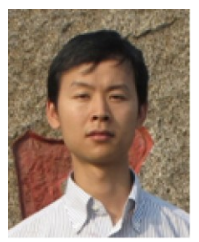

Long Chen received his $\mathrm{PhD}$ degree in 2009 at the Institute for Molecular Science (IMS, Japan). He then joined Max Planck Institute for Polymer Research (MPIP, Germany) as an Alexander von Humboldt research fellow. He joined Tianjin University as a full professor in September, 2014. His current research focuses on the design and synthesis of $2 \mathrm{D}$ conjugated porous polymers for catalysis and energy-related applications.

\section{含钴夹层结构的共价有机框架用于二氧化碳的化学 固定}

李阳 ${ }^{1,2,3}$, 宋肖瑜 ${ }^{3}$, 张广 ${ }^{3}$, 陈卫华 ${ }^{4}$, 王雷 ${ }^{1,2}$, 刘 1 $^{*}$, 陈龙 ${ }^{*}$

摘要 二氧化碳作为主要的温室气体导致了全球变暖, 并威胁着生态 系统的平衡. 有效捕获二氧化碳并将其转化为增值化学品具有重要的 意义，但也充满挑战. 作为一种新兴的多孔晶态材料，共价有机框架 (COFs)不仅能有效吸附气体, 而且可作为化学转化催化剂. 本文报道了 一种由亚胺连接的含夹层钴配合物的 $\mathrm{COF}(\mathrm{Co}-\mathrm{BD}-\mathrm{COF}), \mathrm{Co}-\mathrm{BD}-\mathrm{COF}$ 表现出较高的结晶度和较大的比表面积. Co-BD-COF还兼具丰富的金 属位点和较高的孔隙率, 能有效地催化 $\mathrm{CO}_{2}$ 转化为环状碳酸酯. 此外, Co-BD-COF在环加成反应中表现出对小体积的环氧乙烷衍生物的高 效选择性，这种高效选择性来源于夹层钴配合物周围的苯基部分所形 成的空间位阻作用. 将金属夹层配合物构筑到COF骨架，为提高COFs 的催化选择性提供了一种新的设计策略. 